\title{
MBER Turbo Multiuser Beamforming Aided QPSK Receiver Design Using EXIT Chart Analysis
}

\author{
S. Tan, S. Chen and L. Hanzo \\ School of Electronics and Computer Science \\ University of Southampton, Southampton, SO17 1BJ, U.K. \\ $\{$ st104r,sqc,lh\}@ecs.soton.ac.uk
}

\begin{abstract}
This paper studies the mutual information transfer characteristics of a novel iterative soft interference cancellation (SIC) aided beamforming receiver designed for quadrature phase shift keying (QPSK) modulated systems communicating over additive white Gaussian noise (AWGN) channels. Based on the extrinsic information transfer (EXIT) chart technique, we investigate the convergence behaviour of an iterative minimum bit error rate (MBER) multiuser detection scheme as a function of the system parameters and channel conditions. We also compare the achievable performance and convergence behaviour of different multiuser detectors (MUD) and channel decoders. Our simulation results show that the EXIT chart analysis is sufficiently accurate for reliably predicting the performance of the MBER MUD, despite its potentially non-Gaussian output distribution because we invoke the histogram-based approximation of the true distribution. As expected, the proposed SICMBER MUD outperforms the SIC aided minimum mean square error (SIC-MMSE) MUD.
\end{abstract}

\section{INTRODUCTION}

Since the discovery of turbo codes [1], iterative detection has been applied in the context of joint channel estimation and equalization [2], [3], in multiuser detection [4], [5] and numerous other coded communication systems [6]. Most studies consider the MMSE SIC aided iterative receiver [2]-[5]. However, the MMSE algorithm does not guarantee the direct and explicit minimization of the system's BER. Hence in references [7], [8] the BER, rather than the MSE was minimized at the MUD's output. It is plausible that in terms of the BER performance the MBER beamforming design is the true optimal solution and hence it generally outperforms the MMSE solution, particularly in the context of the so-called rank-deficient systems, where the degree of freedom provided by the antenna array is lower than the number of users.

The concept of EXIT charts was introduced in [9]. This semianalytic technique uses the mutual information between the inputs and outputs of the concatenated receiver components in order to analyze their achievable performance. For example, EXIT charts were employed in turbo equalization in [3], while in [5] they were used for examining the convergence properties of a turbo MUD. Until recently, EXIT chart analysis was only capable of predicting the achievable decoding performance, when the extrinsic information was Gaussian distributed, but Li and Wang [5] succeeded in adopting this technique also in the context encountering a non-Gaussian distribution at the output of a turbo MUD. When the receiver's output distribution cannot be sufficiently accurately modeled by the ubiquitous Gaussian distribution, typically an experimentally recorded output histogram is used for generating the EXIT chart [5]. We also opted for invoking this approach.

The financial support of the EU under the auspices of the Phoenix and Newcom projects as well as that of the EPSRC UK is gratefully acknowledged.
The novel contribution of this treatise is that iterative MBER detection is proposed and its performance is studied with the aid of multi-user EXIT charts, despite the challenge of having a potentially non-Gaussian mutual information distribution at the MUD's output. The structure of this contribution is as follows. In Section II, we outline the signal model used, followed by the portrayal of our iterative beamformer design. The focus of Section III is the novel MBER soft-input soft-output (SISO) interference canceller advocated. Section IV introduces the EXIT chart principles. Our simulation results and EXIT chart analysis are presented in Section V, followed by our conclusions in Section VI.

\section{SYSTEM DESCRIPTION}

\section{A. Signal Model}

The system supports $K$ number of QPSK users and each user transmits his/her signal on the same carrier frequency of $\omega=2 \pi f$. The receiver is equipped with a linear antenna array consisting of $L$ elements, which have a uniform element spacing of $\lambda / 2$, where $\lambda$ is the wavelength. Assume that the channel is non-dispersive in both the angular domain as well as in the time domain and hence does not induce intersymbol interference (ISI). Then the symbol-rate-sampled received signal can be expressed as $r_{l}(n)=$ $\sum_{k=1}^{K} A_{k} s_{k}(n) e^{j \omega t_{l}\left(\theta_{k}\right)}+n_{l}(n)$ for $1 \leq l \leq L$, where $A_{k}$ is the nondispersive complex-valued channel coefficient of user $k, s_{k}(n)$ is the $n$th symbol of the $k$ th QPSK user, while $n_{l}(n)$ is a complex-valued Gaussian white noise process associated with $E\left[\left|n_{l}(n)\right|^{2}\right]=2 \sigma_{n}^{2}$. Furthermore, $t_{l}\left(\theta_{k}\right)=\frac{\lambda}{2}(l-1) \sin \left(\theta_{k}\right) / c$ is the relative time delay at array element $l$ for the source signal of user $k$, with $\theta_{k}$ being the line of sight (LOS) angle of arrival for source $k$, and $c$ is the speed of light. The received signal vector $\mathbf{r}(n)=\left[r_{1}(n) r_{2}(n) \cdots r_{L}(n)\right]^{T}$ is given by $\mathbf{r}(n)=\mathbf{H s}(n)+\mathbf{n}(n)$, where we have $\mathbf{n}(n)=\left[n_{1}(n) n_{2}(n) \cdots n_{L}(n)\right]^{T}$, the transmitted symbol vector of the $K$ users is $\mathbf{s}(n)=\left[s_{1}(n) s_{2}(n) \cdots s_{K}(n)\right]^{T}$ and the system matrix is denoted by $\mathbf{H}=\left[\begin{array}{lll}\mathbf{h}_{1} & \mathbf{h}_{2} \cdots \mathbf{h}_{K}\end{array}\right]$, which is associated with the steering vector $\mathbf{h}_{k}=\left[A_{k} e^{j \omega t_{1}\left(\theta_{k}\right)} A_{k} e^{j \omega t_{2}\left(\theta_{k}\right)} \cdots A_{k} e^{j \omega t_{L}\left(\theta_{k}\right)}\right]^{T}$ of source $k, k=1, \cdots, K$.

\section{B. Iterative Multiuser Beamforming Receiver Structure}

The iterative multiuser beamforming receiver's structure is shown in Fig. 1, which consists of two stages, namely the SISO interference cancellation aided beamforming multiuser detector, followed by $K$ parallel single-user SISO channel decoders. The two stages are separated by the usual bit-based deinterleavers $\Pi^{-1}$ and interleavers $\Pi$.

The proposed SISO beamforming MUD first determines the coefficients of the beamformer weight vector $\mathbf{w}_{k}(n)$ according to the specific design criterion employed and uses this weight vector for 


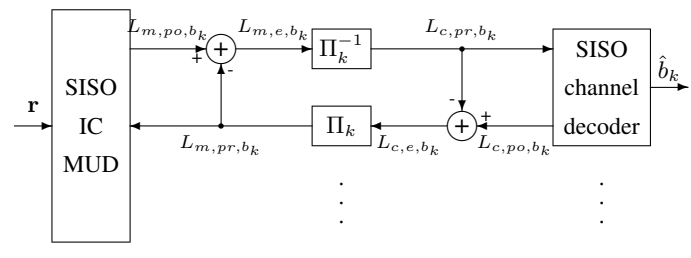

Fig. 1. Iterative multiuser beamforming receiver structure

estimating symbol $\hat{s}_{k}(n)$ corresponding to the transmitted symbol $s_{k}(n)$ from the received signal $\mathbf{r}(n)$ with the aid of a linear transformation. Let us now define $b_{k}(n, i)$ as the $i$ th $(i=1,2)$ bit of the QPSK symbol $s_{k}(n)$, whereas $b_{k}(j)$ is the same bit but in a different position of the bit-based interleaving block after the deinterleaver. We will use the subscripts $m$ and $c$ that are associated with the multiuser detector and channel decoder, respectively, while the subscripts $p r, p o$ and $e$ are used for representing the a priori, a posteriori and extrinsic information. Then the SISO beamforming MUD delivers the a posteriori information of bit $b_{k}(n, i)$ expressed in terms of its log-likelihood ratio (LLR) as [4]

$$
\begin{aligned}
L_{m, p o, b_{k}(n, i)} & =\ln \frac{\mathrm{P}\left[\hat{s}_{k}(n) \mid b_{k}(n, i)=0\right]}{\mathrm{P}\left[\hat{s}_{k}(n) \mid b_{k}(n, i)=1\right]}+\ln \frac{\mathrm{P}\left[b_{k}(n, i)=0\right]}{\mathrm{P}\left[b_{k}(n, i)=1\right]} \\
& =L_{m, e, b_{k}(n, i)}+L_{m, p r, b_{k}(n, i)},
\end{aligned}
$$

where the second term, denoted by $L_{m, p r, b_{k}(n, i)}$, represents the a priori logarithmic likelihood ratio (LLR) of the interleaved and encoded bits $b_{k}(n, i)$. The first term in Equation (1), which is denoted by $L_{m, e, b_{k}(n, i)}$, represents the extrinsic information delivered by the SISO multiuser detector, based on the received signal $\mathbf{r}(n)$ and on the a priori information about the encoded bits of all users, except the $i$ th bit of the desired user $k$. The extrinsic information is then deinterleaved and fed into the $k$ th user's channel decoder, which will provide the a priori information in the next iteration.

As seen in Fig. 1, between the banks of channel decoders and interleavers, we compute the extrinsic LLR based on the a priori information $L_{c, p r, b_{k}(j)}$ provided by the SISO beamforming MUD for the SISO decoder as $L_{c, e, b_{k}(j)}=L_{c, p o, b_{k}(j)}-L_{c, p r, b_{k}(j)}$ [4], where the extrinsic information is gleaned from the surrounding encoded bits, excluding the specific bit considered [4]. After interleaving, the extrinsic information delivered by the channel decoders is fed back to the SISO multiuser detector, as the a priori information concerning the encoded bits of all the users for exploitation during the next iteration.

\section{SISO INTERFERENCE CANCELLATION}

As described in the previous section, the task of SISO interference cancellation is to choose the beamformer weight vector $\mathbf{w}_{k}$ of the linear filter seen in Fig. 1 according to an appropriate design criterion and compute the corresponding output LLRs.

Given the a priori LLRs, we first define the mean and variance of the $k$ th user's symbols for QPSK as in [2], where the symbol-index $n$ was dropped for notational convenience: $\bar{s}_{k}=\left(\tanh \left(\frac{L_{p r, b_{k}(1)}}{2}\right)+\right.$ $\left.j \tanh \left(\frac{L_{p r, b_{k}(2)}}{2}\right)\right) / \sqrt{2}$ and $v_{k}=1-\left(\tanh ^{2}\left(\frac{L_{p r, b_{k}(1)}}{2}\right)+\right.$ $\left.\tanh ^{2}\left(\frac{L_{p r, b_{k}(2)}}{2}\right)\right) / 2$. When using the soft interference cancellation principle, the estimated symbol of user $k$ can be expressed as [2]

$$
\hat{s}_{k}=\mathbf{w}_{k}^{H}\left(\mathbf{r}-\mathbf{H} \overline{\mathbf{s}}+\mathbf{h}_{k} \bar{s}_{k}\right),
$$

where we have $\overline{\mathbf{s}}=\left[\begin{array}{llll}\bar{s}_{1} & \bar{s}_{2} \cdots \bar{s}_{K}\end{array}\right]^{T}$. In the next two sections we will outline the differences of the MBER MUD and the MMSE MUD.

\section{A. SISO Interference Cancellation Using the MMSE MUD}

Classically, the MMSE solution of the beamformer's weight vector $\mathbf{w}_{k}$ is expressed as [2]

$$
\mathbf{w}_{k, m m s e}=\left(\mathbf{H V H}{ }^{H}+\left|\bar{s}_{k}\right|^{2} \mathbf{h}_{k} \mathbf{h}_{k}^{H}+2 \sigma_{n}^{2} \mathbf{I}_{L}\right)^{-1} \mathbf{h}_{k},
$$

where $\mathbf{I}_{L}$ denotes the $(L \times L)$-dimensional identity matrix and we have $\mathbf{V}=\operatorname{diag}\left[v_{1} v_{2} \cdots v_{K}\right]$.

As stated in [2], the conditional probability density function (PDF) $\mathrm{P}\left[\hat{s}_{k} \mid s_{k}=s_{k}^{(q)}\right]$, where $s_{k}^{(q)}$ is the $q$ th $(q=1,2, \cdots, 4)$ legitimate value of the symbol $s_{k}$, may be assumed to be Gaussian distributed and the corresponding extrinsic output LLR is given by [2]

$$
L_{e, b_{k}(1)}=\frac{2 \sqrt{2} \Re\left[\mathbf{w}_{k}^{H}\left(\mathbf{r}-\mathbf{H} \overline{\mathbf{s}}+\bar{s}_{k} \mathbf{h}_{k}\right)\right]}{1-\mathbf{w}_{k}^{H} \mathbf{h}_{k}},
$$

and

$$
L_{e, b_{k}(2)}=\frac{2 \sqrt{2} \Im\left[\mathbf{w}_{k}^{H}\left(\mathbf{r}-\mathbf{H} \overline{\mathbf{s}}+\bar{s}_{k} \mathbf{h}_{k}\right)\right]}{1-\mathbf{w}_{k}^{H} \mathbf{h}_{k}}
$$

for QPSK, where $\Re[\cdot]$ denotes the real part and $\Im[\cdot]$ denotes the imaginary part .

\section{B. SISO Interference Cancellation Using the MBER MUD}

In QPSK systems supporting $K$ users, the error probability of the QPSK-bit constituting the real part is [7]

$$
\mathrm{Pe}_{k, R}\left(\mathbf{w}_{k}\right)=\sum_{q=1}^{4^{K}} \mathrm{P}\left(\mathbf{s}^{(q)}\right) \cdot Q\left[\frac{\operatorname{sgn}\left(\Re\left[s_{k}^{(q)}\right]\right) \cdot \Re\left[\hat{s}_{k}^{(q)}\right]}{\sigma_{n} \sqrt{\mathbf{w}_{k}^{H} \mathbf{w}_{k}}}\right],
$$

where $\mathrm{P}\left(\mathbf{s}^{(q)}\right)=\prod_{k} \mathrm{P}\left(s_{k}=s_{k}^{(q)}\right)$ is the a priori probability of transmitting the $q$ th $\left(q=1,2, \cdots, 4^{K}\right)$ possible symbol combination $\mathbf{s}^{(q)}$ of the $K$ users, $\overline{\hat{s}}_{k}^{(q)}=\mathbf{w}_{k}^{H}\left(\mathbf{H} \mathbf{s}^{(q)}-\mathbf{H} \overline{\mathbf{s}}+\bar{s}_{k} \mathbf{h}_{k}\right)$ is the $k$ th user's estimated symbol, when ignoring the effects of noise, and $Q[x]=(1 / \sqrt{2 \pi}) \int_{-\infty}^{x} e^{-t^{2} / 2} d t$. Similarly, the error probability of the imaginary part is given by

$$
\mathrm{Pe}_{k, I}\left(\mathbf{w}_{k}\right)=\sum_{q=1}^{4^{K}} \mathrm{P}\left(\mathbf{s}^{(q)}\right) \cdot Q\left[\frac{\operatorname{sgn}\left(\Im\left[s_{k}^{(q)}\right]\right) \cdot \Im\left[\overline{\hat{s}}_{k}^{(q)}\right]}{\sigma_{n} \sqrt{\mathbf{w}_{k}^{H} \mathbf{w}_{k}}}\right] .
$$

Hence the MBER beamforming solution is then defined as [7]

$$
\mathbf{w}_{k, m b e r}=\arg \min _{\mathbf{w}_{k}} \frac{1}{2}\left(\mathrm{Pe}_{k, R}+\mathrm{Pe}_{k, I}\right) \text {. }
$$

This optimization problem can be solved using the simplified conjugate gradient algorithm, which is detailed in [7]. The gradients of both the inphase and quadrature-phase bit error probabilities are given by

$$
\begin{gathered}
\nabla \mathrm{Pe}_{k, R}\left(\overline{\mathbf{w}}_{k}\right)=\frac{1}{\sqrt{2 \pi} \sigma_{n}} \sum_{q=1}^{N_{b}} \mathrm{P}\left(\mathbf{s}^{(q)}\right) \cdot \exp \left(-\frac{\left(\Re\left[\overline{\hat{s}}_{k}^{(q)}\right]\right)^{2}}{2 \sigma_{n}^{2}}\right) \\
\cdot \operatorname{sgn}\left(\Re\left[s_{k}^{(q)}\right]\right)\left(\overline{\mathbf{w}}_{k} \Re\left[\overline{\hat{s}}_{k}^{(q)}\right]-\left(\mathbf{H} \mathbf{s}^{(q)}-\mathbf{H} \overline{\mathbf{s}}+\bar{s}_{k} \mathbf{h}_{k}\right)\right)
\end{gathered}
$$

and

$$
\begin{array}{r}
\nabla \mathrm{Pe}_{k, I}\left(\overline{\mathbf{w}}_{k}\right)=\frac{1}{\sqrt{2 \pi} \sigma_{n}} \sum_{q=1}^{N_{b}} \mathrm{P}\left(\mathbf{s}^{(q)}\right) \cdot \exp \left(-\frac{\left(\Im\left[\overline{\hat{s}}_{k}^{(q)}\right]\right)^{2}}{2 \sigma_{n}^{2}}\right) \\
\cdot \operatorname{sgn}\left(\Im\left[s_{k}^{(q)}\right]\right)\left(\overline{\mathbf{w}}_{k} \Im\left[\overline{\hat{s}}_{k}^{(q)}\right]+j\left(\mathbf{H} \mathbf{s}^{(q)}-\mathbf{H} \overline{\mathbf{s}}+\bar{s}_{k} \mathbf{h}_{k}\right)\right),
\end{array}
$$

where $\overline{\mathbf{w}}_{k}$ is the unity-norm normalized version of the vector $\mathbf{w}_{k}$.

The MBER design exploits the fact that both the real part and the imaginary part of the estimated symbols may be non-Gaussian, especially when the number of interferers is relatively low. Hence the 
challenge is that the Gaussian approximation may not be sufficiently accurate for calculating the output extrinsic LLRs of the MBER multiuser detector. The exact expression of the extrinsic information delivered by the MUD is [4]

$$
\begin{aligned}
& L_{e, b_{k}(i)}= \\
& \ln \frac{\sum_{\forall \mathbf{s}^{(q)}: b_{k}^{(q)}(i)=0} \mathrm{P}\left(\hat{s}_{k} \mid \mathbf{s}^{(q)}\right) \prod_{\forall\left(k^{\prime}, i^{\prime}\right) \neq(k, i)} \mathrm{P}\left(b_{k^{\prime}}\left(i^{\prime}\right)^{(q)}\right)}{\sum_{\forall \mathbf{s}^{(q)}: b_{k}^{(q)}(i)=1} \mathrm{P}\left(\hat{s}_{k} \mid \mathbf{s}^{(q)}\right) \prod_{\forall\left(k^{\prime}, i^{\prime}\right) \neq(k, i)} \mathrm{P}\left(b_{k^{\prime}}\left(i^{\prime}\right)^{(q)}\right)},
\end{aligned}
$$

where we have

$$
\mathrm{P}\left(\hat{s}_{k} \mid \mathbf{s}^{(q)}\right)=\frac{1}{2 \pi \sigma_{n}^{2}} \exp \left(-\frac{\left|\overline{\mathbf{w}}_{k}^{H}\left(\mathbf{r}-\mathbf{H s}^{(q)}\right)\right|^{2}}{2 \sigma_{n}^{2}}\right),
$$

which represents the conditional probability of the $k$ th user's estimated symbol, when transmitting the $q$ th $K$-user QPSK symbol combination $\mathbf{s}^{(q)}$. Furthermore $\mathrm{P}\left(b_{k^{\prime}}\left(i^{\prime}\right)^{(q)}\right)=$ $\left(1+\operatorname{sgn}\left(b_{k^{\prime}}\left(i^{\prime}\right)^{(q)}\right) \tanh \left(\frac{L_{p r, b} k^{\prime}\left(i^{\prime}\right)}{2}\right)\right) / 2$ is the probability of the $k^{\prime}$ th user's bit in case of the $q$ th $K$-user QPSK symbol combination quantified in terms of its a priori information [1].

\section{EXIT ChaRt ANALYSIS FOR NON-GAUSSIAN MUtUAL INFORMATION}

The EXIT chart analysis computes the mutual information between the LLRs and the corresponding bits, as detailed in [9]. The EXIT chart is either the nonlinear transfer function $I_{m, o}=f_{m}\left(I_{m, i}, E_{b} / N_{0}\right)$ of the MUD or the corresponding function $I_{c, o}=f_{c}\left(I_{c, i}\right)$ of the channel decoder, which maps the input variable $I_{i}$ to the output variable $I_{o}$, and the specific value of $I_{o}$ in the range $[0,1]$ characterizes the quality of the output LLRs of a receiver component.

The output of one of the constituent detectors is the input of the other, hence both transfer functions are shown in the same EXIT plane having coordinate axes of $\left(I_{m, i}=I_{c, o}\right),\left(I_{m, o}=I_{c, i}\right)$. The staircase-shaped lines connecting the mutual information points evaluated during each iteration are referred to as the detection or decoding trajectory. The substantial advantage of EXIT charts accrues from the fact that the detection trajectory points recorded for both constituent components exchanging information fall on the continuous EXIT functions obtained independently in a separate process. An infinitesimally low BER may be attained, when there is a so-called open tunnel between the EXIT curves of the decoder and the MUD. This graphical representation gives us an immediate insight into the number of detection iterations required for attaining the best possible BER performance.

\section{Simulation Results}

In this section, we use EXIT charts to analyze the attainable performance of the iterative MBER beamforming receiver. The system employs a two-element antenna array. All users employ QPSK modulation and have the same transmit power as well as channel coefficients of $A_{k}=1.0+j 0.0$ (for $1 \leq k \leq K$ ). The angular separation of users with respect to the antenna array are shown in Table I. All users employ the same rate $1 / 2$ and constraint length 4 non-

TABLE I

ARRIVAL ANGLES OF THE USERS' SIGNALS

\begin{tabular}{c|ccccc} 
user number & \multicolumn{5}{|c}{ angles of arrival } \\
$K$ & $\theta_{1}$ & $\theta_{2}$ & $\theta_{3}$ & $\theta_{4}$ & $\theta_{5}$ \\
\hline 3 & $15^{\circ}$ & $-24^{\circ}$ & $68^{\circ}$ & & \\
4 & $15^{\circ}$ & $-48^{\circ}$ & $-14^{\circ}$ & $49^{\circ}$ & \\
5 & $15^{\circ}$ & $-8^{\circ}$ & $41^{\circ}$ & $-33^{\circ}$ & $-70^{\circ}$
\end{tabular}

systematic convolutional (NSC) code using the octally represented generators $(15,17)$. Each user employs a different randomly generated interleaver. The interleaver length of each user is $2 \times 10^{4}$ bits.

\section{A. EXIT-Chart Trajectories of the MBER MUD}

According to the principles outlined in Section IV, in Fig. 2 we plot the EXIT curves of both the MUD (the dotted line) and that of the decoder (the dashed line) along with the simulated trajectories (the solid lines with arrows) of the iterative MBER beamforming receiver supporting $K=4$ users at $E_{b} / N_{0}=2 \mathrm{~dB}, 3 \mathrm{~dB}$ and $4 \mathrm{~dB}$. The detection trajectories closely follow the EXIT curves of the

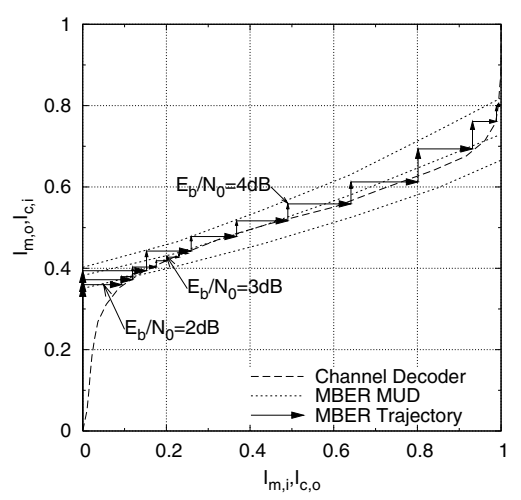

Fig. 2. EXIT charts and simulated trajectories of the iterative MBER receiver supporting $K=4$ users at $E_{b} / N_{0}=2 \mathrm{~dB}, 3 \mathrm{~dB}$ and $4 \mathrm{~dB}$

receiver components, which indicates that the EXIT chart analysis is sufficiently accurate for the MBER MUD. As seen in Fig. 2, at $E_{b} / N_{0}=2 \mathrm{~dB}$ the trajectory is curtailed after two iterations, since the EXIT curves of the MUD and the decoder do intersect. By contrast, at $E_{b} / N_{0}=4 \mathrm{~dB}$, the decoding trajectory passes through "the bottleneck" and reaches the top-right corner, indicating an infinitesimally low BER. We observe that after a few iterations, the trajectories slightly deviate from the EXIT curves, which is a consequence of the extrinsic information becoming correlated upon increasing the number of iterations, in particular, when the interleaving length is finite.

Unlike in single-user turbo coding or turbo equalization, the turbo multiuser detector processes the superposition of all the users' mutual information in order to generate the corresponding 2-dimensional EXIT chart. In our simulations, all users' received signal powers were selected to render the users' SIRs similar. If the users' SIRs are unequal, the averaged EXIT trajectories will deviate from the EXIT transfer curves and consequently the EXIT chart analysis becomes less accurate.

\section{B. Operating SNR Threshold Estimation}

We can infer from the above results that the turbo detection scheme is capable of providing significant performance improvements, when the iterative process converges successfully. However, achieving successful convergence depends upon a number of factors, such as the user load, the type of detector, as well as the channel code and the SNR considered, all of which will be discussed below.

From Fig.2, it is readily seen that if $E_{b} / N_{0}$ is higher than $3 \mathrm{~dB}$, there is an open tunnel between the EXIT curve of the MUD and that of the decoder. The iterative process will hence successfully converge to an infinitesimally low BER. Hence we refer to $E_{b} / N_{0}=3 \mathrm{~dB}$ as the operating SNR threshold of this system.

Fig. 3 shows the BER performance of the system, when increasing the number of iterations. It can be seen that when the SNR is higher 


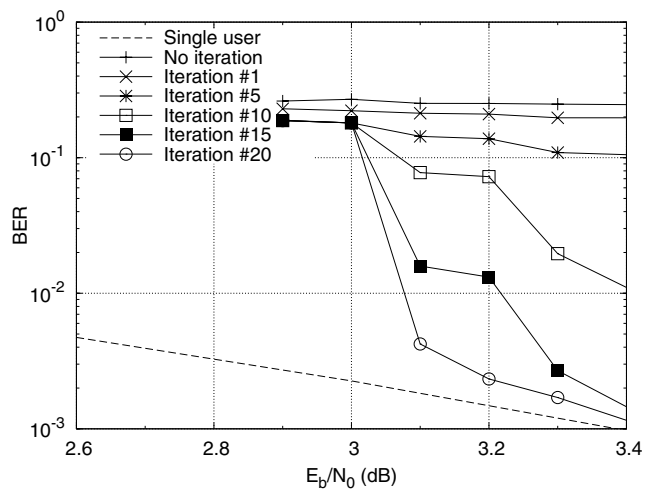

Fig. 3. BER performance of the iterative MBER beamforming receiver supporting $K=4$ users

than $3 \mathrm{~dB}$, the achievable BER approaches that of the single-user bound, which confirms the predictions of the EXIT charts. Finally, it is worth noting that the narrower the EXIT tunnel, the higher the number of iterations required for achieving detection convergence.

\section{The Number of User Supported}

In addition to the operating SNR threshold, there are other thresholds in turbo multiuser detection, which are of interest. For example, given a certain SNR, the EXIT curve of the detector moves downwards upon increasing the number of users $K$, potentially closing the convergence tunnel. This limits the maximum number of users that the system can support at this SNR.

Fig. 4 shows the EXIT curves of the channel decoder and the MBER MUD, when supporting different number of users $K$ at $E_{b} / N_{0}=4 \mathrm{~dB}$. The EXIT chart shows that at $E_{b} / N_{0}=4 \mathrm{~dB}$, the

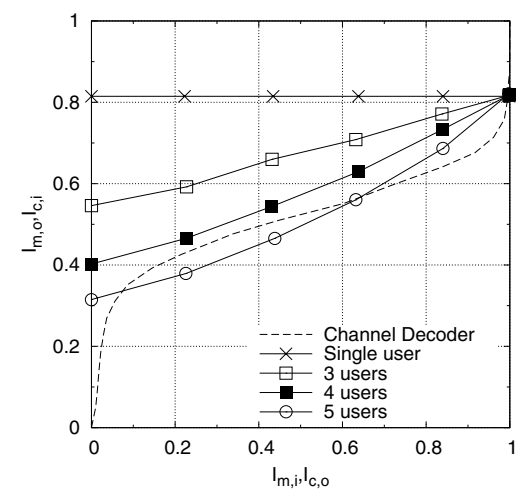

Fig. 4. EXIT charts for the MBER MUD and NSC channel decoder for different number of users at $E_{b} / N_{0}=4 \mathrm{~dB}$

maximum number of users is $K=4$, where an open EXIT-tunnel is visible. It is clear that the maximum number of users supported is a function of the SNR, as well as of the specific detection and decoding schemes employed.

Fig. 4 also shows that all the MUD EXIT curves converge to the ordinate value of $I_{m, o} \approx 0.82$ at the abscissa of $I_{m, i}=1$. This is because regardless of the number of users, when the a priori information is perfect, all the other users' interference can be perfectly removed, resulting in a near-single-user performance. We also note that the point of perfect convergence at $(1,1)$ is not reached, since the BER performance of the MUD depends almost purely on the SNR, when the multiuser interference (MUI) has been perfectly removed. When the SNR is infinitely high, the point of $(1,1)$ can indeed be reached.

\section{Comparison of Different Turbo-MUDs}

Consider a four-user system employing two receive antennas. Fig. 5 shows the EXIT characteristics of the iterative MUDs employing both the MBER and the MMSE detection schemes operating at $E_{b} / N_{0}=2 \mathrm{~dB}, 3 \mathrm{~dB}$ and $4 \mathrm{~dB}$, both of which have a similar EXIT tunnel. Fig. 5 also reveals that the two detectors yield the same

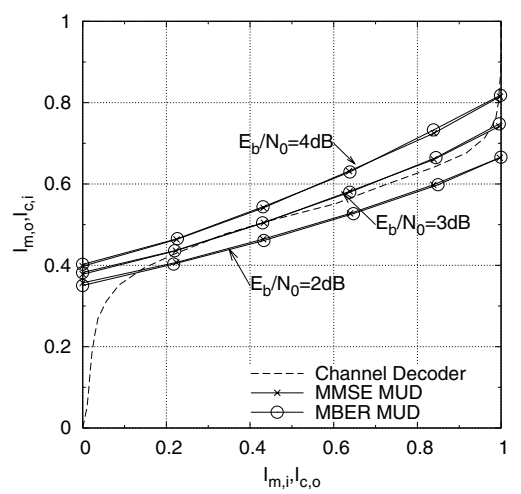

Fig. 5. EXIT characteristics of the different SISO MUDs supporting $K=4$ users at $E_{b} / N_{0}=2 \mathrm{~dB}, 3 \mathrm{~dB}$ and $4 \mathrm{~dB}$

value of $I_{m, o}<1$ with the advent of perfect a priori information corresponding to $I_{m, i}=1$. This is because for $\left|L_{m}\right| \rightarrow \infty$, the multiple access interference (MAI) can be completely removed from the received signal.

The main difference between the two detectors is the slope of the EXIT curves observed in Fig. 5, which is quite small for QPSK systems and will still affect both the SNR convergence threshold and the convergence rate of the associated turbo receiver. Fig. 6 shows the BER versus SNR performance of these two MUD algorithms. The SNR thresholds are $3.1 \mathrm{~dB}$ and $3.3 \mathrm{~dB}$ for the MBER and

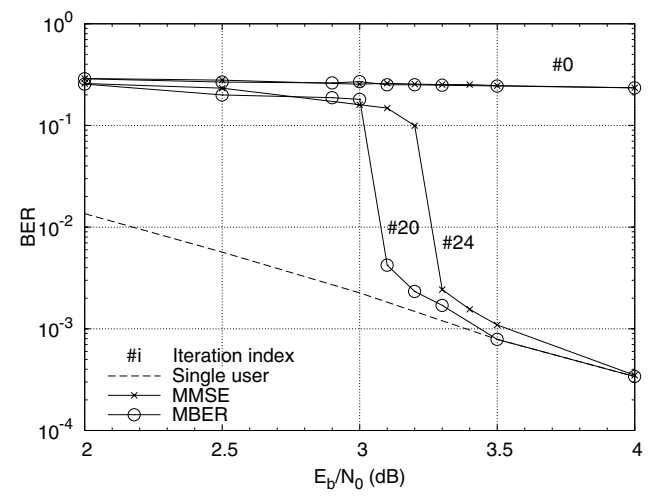

Fig. 6. BER comparison of the MMSE and MBER iterative MUDs supporting $K=4$ users

MMSE MUDs, respectively. It can be seen that the performance of both beamforming receivers has significantly improved after $i=20$ iterations and $i=24$ iterations, respectively. In this rank-deficient system, namely when the channel-matrix becomes rank-deficient and non-invertible, supporting twice the number of users in comparison to the number of antennas, the MBER algorithm has the lower operating SNR requirement. 


\section{E. Comparison of Different Channel Coding Schemes}

Let us now compare the performance of the MBER turbo receivers using different channel codes, namely the previously used NSC code and a recursive systematic convolutional (RSC) code. Both codes have the same code rate of $1 / 2$ and constraint length 4 . The generator polynomials are $(15,17)$ and $(13,6)$ in octal representation, respectively.

Fig. 7 shows the EXIT charts of the NSC and RSC channel decoders along with the MBER MUD characteristics, when supporting $K=4$ users at $E_{b} / N_{0}=2 \mathrm{~dB}, 3 \mathrm{~dB}$ and $4 \mathrm{~dB}$. In Fig. 7, the arrows

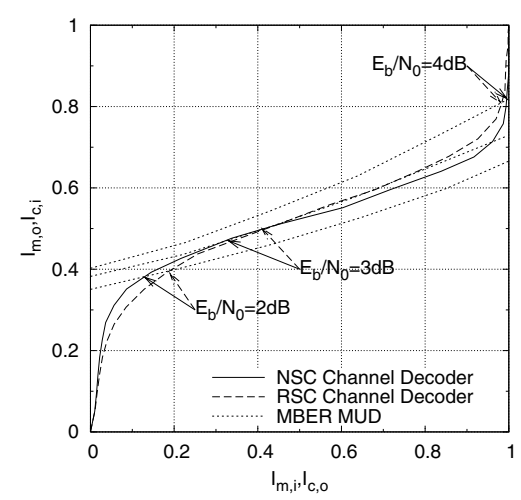

Fig. 7. EXIT charts of the NSC and RSC channel decoders along with the MBER MUD's EXIT characteristics, when supporting $K=4$ users at $E_{b} / N_{0}=2 \mathrm{~dB}, 3 \mathrm{~dB}$ and $4 \mathrm{~dB}$

indicate the intercept points of the channel decoders and the MBER MUD at different SNRs. When the SNR is low, for example $2 \mathrm{~dB}$, the intercept points of both decoders are near the bottom-left corner, with that of the NSC being nearer. This implies that the receiver using the RSC code has a better performance at low SNRs. When the SNR increases to $4 \mathrm{~dB}$, each code provides an open EXIT tunnel and the intercept point of the NSC decoder is moved nearer to the top-right corner than that of the RSC scheme. Hence the NSC receiver performs better. Fig. 8 shows the simulated BER performance of the NSC and RCS receivers after $i=20$ iterations and $i=17$ iterations, respectively, which confirms the above EXIT-chart based conclusions.

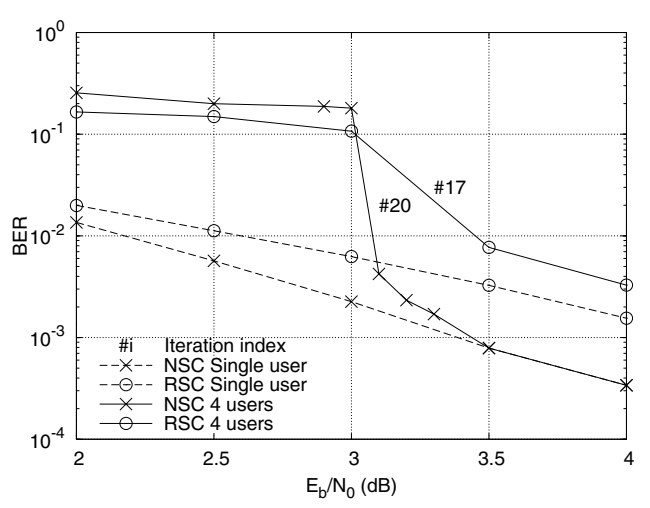

Fig. 8. BER comparison of the NSC and RSC coded MBER turbo receivers supporting $K=4$ users after $i=20$ iterations and $i=17$ iterations, respectively

Observe from Fig. 8 that the NSC-coded receiver has a steep BER curve, while the RSC receiver's BER curve is quite gently sloping. The reason for this difference can be explained by their EXIT charts.
Comparing the EXIT characteristics of the NSC decoder and the MBER MUD at 3dB in Fig. 7, we also observe that the EXIT chart slope of the MUD is slightly steeper than that of the NSC decoder. This implies that the bottleneck is at the left end of the tunnel. When the EXIT tunnel becomes just opened, the receiver becomes capable of achieving a significant BER versus SNR gain, which results in a steep BER curve. By contrast, for the RSC code the slope of the decoder's EXIT curve is steeper than that of the MUD and hence the EXIT-chart intercept point moves more gradually upon increasing the SNR. Hence the BER curve of the RSC receiver does not exhibit the same waterfall phenomenon.

\section{CONCLUSION}

A novel iterative MBER MUD was proposed. Despite the nonGaussian distribution of the mutual information recorded in this multi-user scenario, we succeeded in adopting the classic single-user EXIT-chart concept for our convergence analysis in conjunction with experimentally generated histogram-based estimates of the MUD's output PDF. More explicitly, based on the EXIT charts of the SISO multiuser detectors, the exchange of extrinsic information between the multiuser detectors and the channel decoders was visualized, which facilitated their convergence analysis in the context of iterative detection. EXIT charts were also used for estimating the BER performance of the system at different user loads. Hence our results demonstrate that the EXIT chart can also be used for the analysis of the iterative MBER receiver, whose extrinsic information distribution at the MUD's output is non-Gaussian. Finally, EXIT charts were also used for comparing the convergence behaviour of various turbo receivers using different MUDs and channel codes. Our future research will consider QAM-based MBER MUDs and using irregular-construction channel codes for achieving better convergence properties [10].

\section{REFERENCES}

[1] C. Berrou, A. Glavieux, and P. Thitimajshima, "Near Shannon limit error correcting coding and decoding: Turbo codes," in Proceeding of IEEE International Conference on Communications, Geneva, Switzerland, Vol.2, May 1993, pp.1064-1070

[2] M. Tüchler, A. C. Singer, and R. Koetter, "Minimum mean squared error equalization using a priori information," IEEE Transactions on Signal Processing, Vol.50, No.3, March 2002, pp.673-6820

[3] M. Tüchler, R. Koetter, and A. C. Singer, "Turbo equalization: Principles and new results," IEEE Transactions on Communications, Vol.50, No.5, pp.754-767, May 2002

[4] X. Wang and H. V. Poor, "Iterative (Turbo) soft interference cancellation and decoding for coded CDMA," IEEE Transactions on Communications, Vol.47, No.7, July 1999, pp.1046-1060

[5] K. Li and X. Wang, "EXIT chart analysis of turbo multiuser detection", IEEE Transactions on Wireless Communications, Vol.4, No.1, January 2005, pp.300-311

[6] X. Li and J. Ritcey, "Trellis-coded modulation with bit interleaving and iterative decoding," IEEE Journal on Selected Areas in Communications, Vol.17, pp.715-724, Apr. 1999

[7] S. Chen, N. N. Ahmad, and L. Hanzo, "Adaptive minimum bit error rate beamforming," IEEE Transactions on Wireless Communications, Vol.4, No.2, March 2005, pp.341-348

[8] M. Y. Alias, S. Chen, and L. Hanzo, "Multiple-antenna-aided OFDM employing genetic-algorithm-assisted minimum bit error rate multiuser detection," IEEE Transactions on Vehicular Technology, Vol.54, No.5, September 2005, pp.1713-1721

[9] S. ten Brink, "Convergence behavior of iteratively decoded parallel concatenated codes," IEEE Transactions on Communications, Vol.40, October 2001, pp.1727-1737

[10] J. Wang, S. X. Ng, A. Wolfgang, L. L. Yang, S. Chen and L. Hanzo, "Near-capacity three-stage MMSE turbo equalization using irregular convolutional codes," In Proceedings of Turbo-Coding-2006, Munich, Germany. 